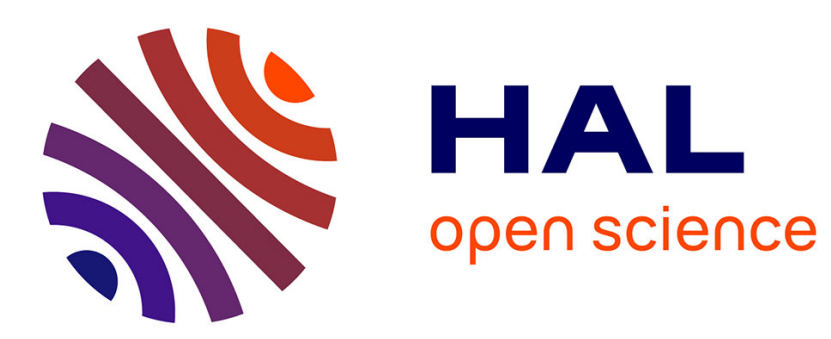

\title{
Collisions between counter-rotating solitary vortices in the three-dimensional Ginzburg-Landau equation
}

Dumitru Mihalache, D. Mazilu, F. Lederer, Hervé Leblond, Boris Malomed

\section{To cite this version:}

Dumitru Mihalache, D. Mazilu, F. Lederer, Hervé Leblond, Boris Malomed. Collisions between counter-rotating solitary vortices in the three-dimensional Ginzburg-Landau equation. Physical Review E: Statistical, Nonlinear, and Soft Matter Physics, 2008, 78 (5), pp.056601. 10.1103/PhysRevE.78.056601 . hal-03423793

\section{HAL Id: hal-03423793 \\ https://univ-angers.hal.science/hal-03423793}

Submitted on 10 Nov 2021

HAL is a multi-disciplinary open access archive for the deposit and dissemination of scientific research documents, whether they are published or not. The documents may come from teaching and research institutions in France or abroad, or from public or private research centers.
L'archive ouverte pluridisciplinaire HAL, est destinée au dépôt et à la diffusion de documents scientifiques de niveau recherche, publiés ou non, émanant des établissements d'enseignement et de recherche français ou étrangers, des laboratoires publics ou privés. 


\title{
Collisions between counter-rotating solitary vortices in the three-dimensional Ginzburg-Landau equation
}

\author{
D. Mihalache, ${ }^{1}$ D. Mazilu, ${ }^{1}$ F. Lederer, ${ }^{2}$ H. Leblond, ${ }^{3}$ and B. A. Malomed ${ }^{4}$ \\ ${ }^{1}$ Horia Hulubei National Institute for Physics and Nuclear Engineering (IFIN-HH), 407 Atomistilor, \\ Magurele-Bucharest, 077125, Romania \\ ${ }^{2}$ Institute of Solid State Theory and Theoretical Optics, Friedrich-Schiller Universität Jena, Max-Wien-Platz, 1, D-07743 Jena, Germany \\ ${ }^{3}$ Laboratoire POMA, FRE 2988, Université d'Angers, 2 Bd Lavoisier, 49000 Angers, France \\ ${ }^{4}$ Department of Physical Electronics, School of Electrical Engineering Faculty of Engineering, Tel Aviv University, Tel Aviv 69978, Israel
}

(Received 15 June 2008; published 7 November 2008)

\begin{abstract}
We report results of collisions between coaxial vortex solitons with topological charges $\pm S$ in the complex cubic-quintic Ginzburg-Landau equation. With the increase of the collision momentum, merger of the vortices into one or two dipole or quadrupole clusters of fundamental solitons (for $S=1$ and 2, respectively) is followed by the appearance of pairs of counter-rotating "unfinished vortices," in combination with a soliton cluster or without it. Finally, the collisions become elastic. The clusters generated by the collisions are very robust, while the "unfinished vortices," eventually split into soliton pairs.
\end{abstract}

DOI: 10.1103/PhysRevE.78.056601

PACS number(s): 05.45.Yv

\section{INTRODUCTION}

The different types of complex Ginzburg-Landau (CGL) equations are universal models for the description of pattern formation in nonlinear dissipative media [1]. Optical settings, such as laser cavities, provide for important implementations of various types of the CGL equations [2-4]. A physically significant class of patterns produced by these models in two and three dimensions (2D and 3D) are localized vortices, i.e., dissipative solitons with embedded vorticity. Stabilization of the vortices is a challenging problem, as azimuthal perturbations tend to split them [5-7].

A paradigmatic model of this type, which was introduced by Petviashvili and Sergeev [8] exactly with the purpose of modeling 2D localized vortices, and has later drawn a great deal of attention-first of all, in its 1D variant [9] -is the CGL equation with the cubic-quintic (CQ) nonlinearity. Nonlinear optical media that feature the CQ response, in a combination with nonlinear loss (two-photon absorption), include chalcogenide glasses [10] and organic materials [11]. In addition to these solid-state media, optical nonlinearity of the same type was predicted [12] and observed [13] in aqueous colloids, and was also reported in dye solutions [14].

In the framework of the CQ CGL equations in two dimensions, stable solitary vortices (alias vortex solitons), with topological charge (vorticity) $S=1$ and 2, were constructed in works [15], and their 3D counterparts for $S=1,2$, and 3 have been obtained in Refs. $[16,17]$. The stability analysis for those solutions was based on the computation of stability eigenvalues, and verified in direct simulations of the evolution of perturbed vortices. 3D complexes including a vortical component were found too, as solutions to equations of the CGL type [18].

Once stable 3D solitons are available, a problem of straightforward interest is to consider collisions between them. Recently [19], we investigated collisions between corotating $\left(S_{1}=S_{2} \equiv S\right)$ 3D solitary vortices in the coaxial configuration, with the vorticity assuming values $S=1$ and 2 . The starting point was the three-dimensional CQ CGL equa- tion in the general form, see, e.g., Refs. $[16,17,20]$,

$$
\begin{aligned}
i U_{z} & +\left(\frac{1}{2}-i \beta\right)\left(U_{x x}+U_{y y}\right)+\left(\frac{D}{2}-i \gamma\right) U_{t t} \\
& +\left[i \delta+(1-i \varepsilon)|U|^{2}-(\nu-i \mu)|U|^{4}\right] U=0 .
\end{aligned}
$$

In term of optical cavities, $U(z, x, y, t)$ is the local amplitude of the electromagnetic wave in the bulk medium which propagates along axis $z$. The transverse coordinates are $x$ and $y$, while the temporal variable is $t=T-z / V_{0}$, where $T$ is time and $V_{0}$ the group velocity of the carrier wave. The coefficients which are scaled to be $1 / 2$ and 1 in Eq. (1) account, respectively, for diffraction in the transverse plane and the self-focusing Kerr nonlinearity, coefficients $\delta, \varepsilon$, and $\mu$ represent, respectively, the linear loss, cubic gain, and quintic loss, while $\beta \geqslant 0$ is the effective diffusivity in the transverse plane. The latter coefficient appears in models of laser cavities, where it combines the dephasing of the polarization in the dielectric medium, cavity loss, and detuning between the cavity's and atomic frequencies [3].

Further, the coefficient $\nu \geqslant 0$ in Eq. (1) accounts for the self-defocusing quintic nonlinearity, that may compete with the cubic term, according to the experimental observations [10-14], $D$ is the group-velocity dispersion (GVD) coefficient, with $D>0$ and $D<0$ corresponding, respectively, to the anomalous and normal GVD, and $\gamma \geqslant 0$ accounts for the spectral filtering (alias dispersion of the linear loss). In both the $2 \mathrm{D}$ or $3 \mathrm{D}$ nonlinear Schrödinger equation with a $\mathrm{CQ}$ nonlinearity, the quintic term must be self-defocusing, to arrest the collapse induced by the self-focusing cubic term $[6,21]$. However, the self-defocusing sign of the quintic term is not necessary in the case of the CGL equation, because the collapse is prevented by the stronger effect of the quintic loss [16]. In fact, the experimentally observed quintic nonlinearity in the above-mentioned colloidal media may indeed be the self-focusing [13]. The sign of $D$ is not crucial either, because the existence of stable fundamental and vortical 3D dissipative solitons was demonstrated for both anomalous [16] and normal [17] GVD, either case being relevant to 
optics (in the case of $D<0$, the soliton develops a phase chirp along the temporal direction).

In Ref. [19], Eq. (1) was taken with $\gamma=0$, which admits free motion of solitons along axis $z$, generated by the application of a "kick," i.e., multiplication of a quiescent solution by $\exp (-i \chi t)$. In fact, the natural size of $\gamma$ is very small indeed, unless the filtering is enhanced by optical filters inserted into the cavity. The mobility of the solitons opens the way to study collisions between them, setting two solitons in motion by means of the application of the kicks of opposite signs to them [22]. On the contrary to that, free motion in the $(x, y)$ plane is impeded by the "diffusion" term in Eq. (1) with $\beta>0$. As shown earlier $[16,17]$, this term is necessary for the stability of localized vortices, while fundamental solitons, with $S=0$, are stable at $\beta=0$ as well; both the fundamental solitons and vortices may be stable at $\gamma=0$.

The coaxial geometry considered in Ref. [19] made systematic investigation of collisions between corotating solitary vortices practically feasible, due to the axial symmetry of the configuration. Note that the numerical integration of Eq. (1) was performed in Cartesian coordinates, hence potentially dangerous small perturbations which might break the axial symmetry were included in the numerical analysis. Depending on the collision momentum (kick), $\chi$, three generic outcomes were observed: Merger of the corotating vortices into a single one at small $\chi$; quasielastic interaction at large $\chi$; and creation of an extra solitary vortex ("soliton birth" in terms of Ref. [23]) in an intermediate region.

The subject of the present work is a natural extension of the analysis of the case of collisions between "counterrotating" vortex solitons, i.e., ones with opposite vorticities, $S_{1}=-S_{2}=1$ and 2 . Because collisions between solitary vortices and antivortices in the 3D space have never been studied before, in this paper we limit the consideration to the most tractable coaxial configuration, similar to that considered in Ref. [19]. The results, produced by systematic simulations of the collisions in the framework of 3D equation (1), are summarized in Sec. II. At small values of $\chi$, slow collisions are inelastic, leading to a merger of the vortices into one or two clusters of fundamental solitons (dipoles or quadrupoles in the cases of $S= \pm 1$ and $S= \pm 2$, respectively). In the case when two clusters are generated by the collision, they feature decelerating rotation in opposite directions. With the increase of $\chi$, two "unfinished vortices" (counter-rotating multihumped objects without a through hole in the center) emerge from the collision; at intermediate values of $\chi$, they appear along with a cluster of fundamental solitons. As a matter of fact, the "unfinished vortices" replace the original solitary vortices. Finally, the collision becomes elastic at large values of $\chi$. We also explore the post-collision dynamics of the emerging objects. The dipolar and quadrupolar clusters feature very slow expansion, being robust against strong perturbations. On the contrary, the "unfinished vortices" eventually split into dipolar pairs of fundamental solitons.

It is relevant to stress that, at all considered values of the parameters, the collision of counter-rotating vortices never resulted in their complete annihilation (decay into zero). This observation demonstrates that the opposite vorticities carried by the colliding solitons provide for a barrier separating the dynamical regime from falling into the attraction basin of the zero solution. Indeed, all the outcomes of the collisions feature a signature of the initial vorticities.

\section{COLLISIONS BETWEEN COUNTER-ROTATING VORTEX SOLITONS}

\section{A. Settings}

Stationary localized solutions to Eq. (1) are sought for in the usual form,

$$
U(z, x, y, t)=\psi(r, t) \exp (i k z+i S \theta),
$$

where $r$ and $\theta$ are the polar coordinates in the $(x, y)$ plane, $S \geqslant 0$ and $k$ are the integer vorticity and real wave number, and the complex function $\psi(r, t)$ obeys the equation

$$
\begin{aligned}
& \left(\frac{1}{2}-i \beta\right)\left(\psi_{r r}+\frac{1}{r} \psi_{r}-\frac{S^{2}}{r^{2}} \psi\right)+\frac{D}{2} \psi_{t t} \\
& +\left[i \delta+(1-i \varepsilon)|\psi|^{2}-(\nu-i \mu)|\psi|^{4}\right] \psi=k \psi,
\end{aligned}
$$

where we set $\gamma=0$, as said above. Localized solutions to Eq. (3) must decay as $r^{|S|}$ at $r \rightarrow 0$, and exponentially at $r,|t|$ $\rightarrow \infty$. A family of stable solitary-vortex solutions to Eq. (1) was constructed in Ref. [19], by dint of direct simulations of the radial equation, which was obtained by the substitution of $U(z, x, y, t)=\Psi(z, r, t) \exp (i S \theta)$ in Eq. (1) (with $\gamma=0)$, i.e.,

$$
\begin{aligned}
i \Psi_{z} & +\left(\frac{1}{2}-i \beta\right)\left(\Psi_{r r}+\frac{1}{r} \Psi_{r}-\frac{S^{2}}{r^{2}} \Psi\right)+\frac{D}{2} \Psi_{t t} \\
& +\left[i \delta+(1-i \varepsilon)|\Psi|^{2}-(\nu-i \mu)|\Psi|^{4}\right] \Psi=0 .
\end{aligned}
$$

Stationary solutions were found as attractors of this equation, generated by the evolution of input pulses $\Psi(0, r, t)$ $=A r^{|S|} \exp \left[-(1 / 2)\left(r^{2} / \rho^{2}+t^{2} / \tau^{2}\right)\right]$, with constants $A, \rho$, and $\tau$ [19].

As well as in work [19], that was dealing with collisions between corotating vortex solitons, a generic situation for the collisions between counter-rotating ones may be adequately represented by fixing parameter values $D=1$ (which corresponds to anomalous GVD), $\mu=1, \nu=0.1, \delta=0.4, \beta=0.5$, and $\varepsilon=2.3$. In this case, the numerical analysis performed in Ref. [19] yields stable solitary vortices with propagation constants [defined as per Eq. (2)] $k=0.50040$ for $S= \pm 1$, and $k=0.50387$ for $S= \pm 2$. They are also characterized by values of their norm, alias total energy, in terms of optics,

$$
E \equiv 2 \pi \int_{0}^{\infty} r d r \int_{-\infty}^{+\infty} d t|\psi(r, t)|^{2},
$$

which takes the values $E(S=1) \approx 171$ and $E(S=2) \approx 310$. Note that, while the above-mentioned propagation constants for $S=1$ and 2 take close values, the respective energies are broadly different, which is typical to stable vortex solitons in models with the CQ nonlinearity [6].

Thus, to simulate the collisions between counter-rotating solitary vortices, we started, at $z=0$, with a pair of stable solitary vortices in the form of $\psi(r, t+T / 2) \exp (i S \theta)$ and $\psi(r, t-T / 2) \exp (-i S \theta)$, with $S=1$ or 2 , which are separated by a large initial temporal distance, $\Delta t=T$. In most cases, we took $T=30$, but varying the initial separation did not affect outcomes of the collisions. 
As mentioned above, the vortices are set in motion by kicking them in the opposite directions along the common axis, i.e., multiplying each one by $\exp ( \pm i \chi t)$. Thus, the full initial configuration $U(0, x, y, t)$ was

$$
\begin{aligned}
U(0, x, y, t)= & \psi(r, t+T / 2) \exp (i S \theta+i \chi t) \\
& +\psi(r, t-T / 2) \exp (-i S \theta-i \chi t) .
\end{aligned}
$$

Because, at $\gamma=0$, Eq. (1) is Galilean invariant in the longitudinal (i.e., axial) direction, the application of the kick to an isolated quiescent soliton, $U_{0}(z, t, x, y)$, generates an exact solution in the form of a "walking" one,

$$
U_{\chi}(z, x, y, t)=U_{0}(z, x, y, t \mp a \chi) \exp \left( \pm i \chi t-i a \chi^{2} z / 2\right) .
$$

The numerical integration of Eq. (1) was carried out by means of a full 3D implicit (Crank-Nicolson) finitedifference scheme, with typical transverse and longitudinal step sizes $\Delta x=\Delta y=\Delta t=0.2$ and $\Delta z=0.01$ (it was checked that reducing the step sizes did not affect the results). The nonlinear finite-difference equations generated by the scheme were solved with the help of the Picard iteration method, and the resulting linear system was then treated by means of the Gauss-Seidel iterative procedure. To achieve good convergence, 10 Picard and four Gauss-Seidel iterations were generically sufficient. In most cases, a set of 601 discretization points for $t$ was used, while the number of the mesh points for $x$ and $y$, that provided for the good accuracy, slightly depended on the vorticity: $193 \times 193$ for $S=1$, and $201 \times 201$ for $S=2$.

\section{B. Outcomes of the collisions}

Results of collisions between the counter-rotating solitary vortices can be summarized following a gradual increase of the collision momentum (kick strength) $\chi$ in expression (6). The first generic outcome was the merger of the colliding vortices with $\left(S_{1}, S_{2}\right)=(+1,-1)$ into a cluster composed of two fundamental solitons (ones with $S=0$ ), at smallest values of $\chi$, or two such clusters, at larger values, see typical examples in Figs. 1(b) and 1(c), respectively. Similarly, the collision in the case of $\left(S_{1}, S_{2}\right)=(+2,-2)$, results, at smallest or somewhat larger values of $\chi$, into the merger of solitary vortices into one or two clusters composed of four fundamental solitons, see Figs. 2(b) and 2(c). In the case when two clusters emerge from the collision, they feature slow counterrotation, which is gradually decelerated by the effective diffusion included in the model [term $\sim \beta$ in Eq. (1)]. On the contrary, the cluster does not rotate when it is single.

It is worthy to notice that the soliton clusters emerging from the collision clearly break the axial symmetry of the initial configuration; at the same time, as well as the initial localized vortices, all clusters lie in planes $(x, y)$ (alias $t$ $=$ const), which are oriented perpendicular to the axis. In particular, the two-soliton clusters displayed in Fig. 1(c) lie in planes $t=\mp 30$, whereas the four-soliton clusters in Fig. 2(c) lie in planes $t=\mp 33.4$. To characterize the shape of the clusters more accurately, it is relevant to additionally mention that, in Fig. 1(c), coordinates of the centers of the two solitons which form the cluster in plane $t=-30$ are

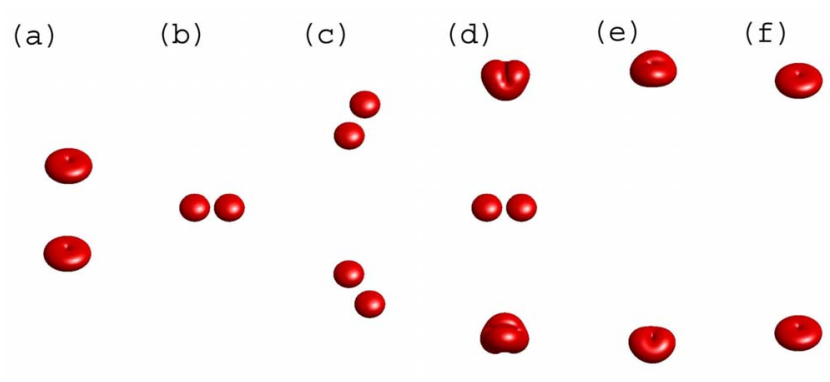

FIG. 1. (Color online) Generic outcomes of collisions between 3D solitary vortices with $\left(S_{1}, S_{2}\right)=(+1,-1)$ are shown by means of isosurface plots of local intensity $|U(x, y, t)|^{2}$, for different values of kick $\chi$. (a) The input configuration (at $z=0$; the localized vortices move, towards their collision, along their common axis, i.e., in the positive and negative vertical directions). (b) A single nonrotating dipole cluster composed of two fundamental solitons, which is the outcome for $\chi=1$ (shown at $z=100$ ). (c) Two counter-rotating dipole clusters, for $\chi=1.5$ (at $z=170$ ); both dipoles lie in planes $t$ $= \pm 30$ oriented perpendicular to the original axis. (d) Two counterrotating double-humped "unfinished vortices," plus a single dipole cluster, for $\chi=2$ (at $z=34$ ). (e) Two counter-rotating "unfinished vortices," without the additional cluster, for $\chi=2.4$ (at $z=27$ ). (f) An elastic collision, for $\chi=4$ (shown at $z=15$ ).

$$
\{(x, y)\}_{\mathrm{two}}^{(-)}=(-3.6,2.0), \quad(3.6,-2.0),
$$

while in plane $t=+30$ they are

$$
\{(x, y)\}_{\mathrm{two}}^{(+)}=(-3.6,-2.0), \quad(3.6,2.0) .
$$

Further, in Fig. 2(c), the centers of the four solitons which form the clusters in planes $t=\mp 33.4$ are located, respectively, at

$$
\{(x, y)\}_{\text {four }}^{(-)}=(-6.0,0.8), \quad(0.8,6.0),
$$

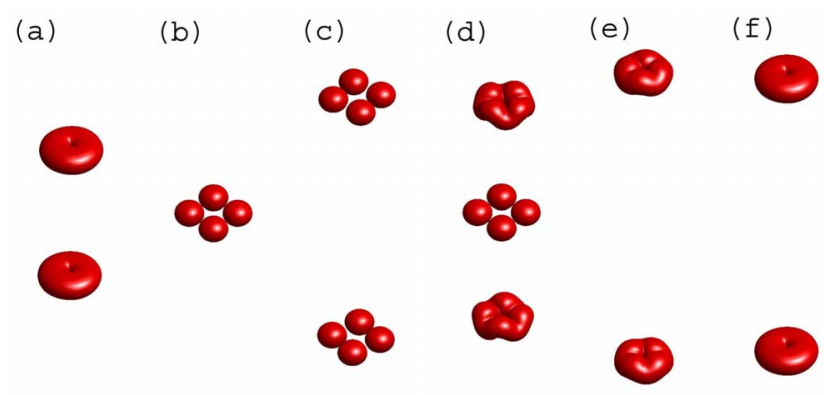

FIG. 2. (Color online) The same as in Fig. 1, but for the collision of solitary vortices with $\left(S_{1}, S_{2}\right)=(+2,-2)$. (a) The input at $z$ $=0$ (as well as in Fig. 1, the localized vortices move, towards their collision, in the vertical directions). (b) A single nonrotating quadrupole cluster composed of four fundamental solitons, which is the outcome of the collisions for $\chi=0.2$ (shown at $z=120$ ). (c) Two counter-rotating quadrupole clusters, for $\chi=0.5$ (at $z=120$ ); the clusters lie in planes $t= \pm 33.4$ oriented perpendicular to the original axis. (d) Two counter-rotating four-humped "unfinished vortices" plus a single quadrupole cluster, for $\chi=1$ (at $z=120$ ). (e) Two counter-rotating "unfinished vortices," without the additional cluster, for $\chi=2.5$ (at $z=25$ ). (f) A quasielastic collision, for $\chi=4$ (shown at $z=16$ ). 

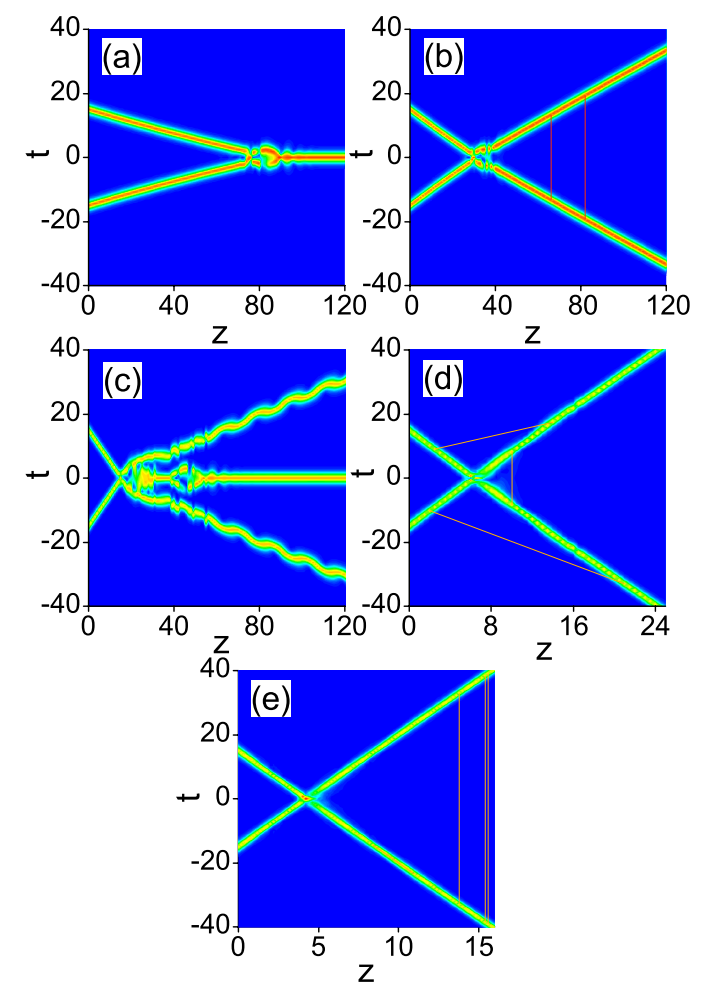

FIG. 3. (Color online) Contour plots display the evolution of $|U|^{2}$ in plane $(t, z)$, for the five scenarios of the collision of solitary vortices with $\left(S_{1}, S_{2}\right)=(+2,-2)$ at different values of kick $\chi$, cf. Fig. 2. (a) The merger into a quadrupole cluster, at $\chi=0.2$. (b) The creation of two quadrupole clusters, at $\chi=0.5$. (c) The appearance of a single cluster and two counter-rotating "unfinished vortices," at $\chi$ $=1$. (d) Two counter-rotating "unfinished vortices," at $\chi=2.5$. (e) The quasielastic collision, at $\chi=4$.

$$
\begin{gathered}
(6.0,-0.8),(-0.8,-6.0), \\
\{(x, y)\}_{\text {four }}^{(+)}=(-6.0,-0.8), \quad(-0.8,6.0), \\
(6.0,0.8), \quad(0.8,-6.0) .
\end{gathered}
$$

The comparison of strings (8) and (9), as well as (10) and (11), demonstrates that the clusters in planes $t<0$ and $t>0$ are obtained from each other by the reflection with respect to axis $x$ or $y$ [such pairs of mutually symmetric clusters were selected for the display in Figs. 1(c) and 2(c) at an appropriate value of evolution variable $z$ ].

In fact, the two-soliton and four-soliton clusters are dipoles and quadrupoles, respectively, as the wave fields in adjacent solitons always have opposite signs. The dipolar and quadrupolar arrangements of the clusters are natural counterparts of the phase structure of the input vortices, with $S= \pm 1$ and \pm 2 , respectively.

Further increase of $\chi$ demonstrates a trend to the transition to elastic collisions. At an intermediate stage, the collision gives rise to a pair of coaxial counter-rotating localized objects, that seem as double-or four-humped bound states of fundamental solitons, in the case of the collisions with $\left(S_{1}, S_{2}\right)=(+1,-1)$ and $(+2,-2)$, respectively. In fact, they may be regarded as "unfinished vortices" (i.e., not "fully (a)

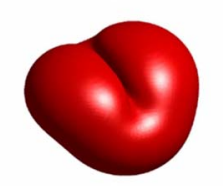

(c)

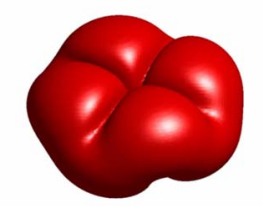

(b)
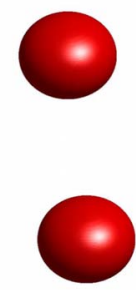

(d)

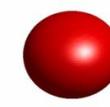

FIG. 4. (Color online) Isosurface plots of intensity $|U(x, y, t)|^{2}$, showing the splitting of the double- and four-humped rotating "unfinished vortices." (a) The input taken from Fig. 1(d) at $z=90$, and (b) the respective eventual set of two fundamental solitons at $z$ $=278$. (c) The input from Fig. 2(e) at $z=110$, and (d) the eventual state at $z=310$. The simulations were run on the grid of size 193 $\times 193 \times 601$ for (a) and (b), and $201 \times 201 \times 601$ for (c) and (d).

processed" ones, without the through hole at the center). The rotation velocities of these objects decrease as long as they exist; this feature may be expected because the dynamical model (1) contains a diffusion term (proportional to the diffusivity parameter $\beta$ ), which should gradually suppress the rotation in the $(x, y)$ plane. The rotation of the pair of "unfinished vortices" is a signature of the presence of the angular momentum, which cannot be absorbed into the intrinsic vorticity, as the shape of the objects does not allow it. In this situation, the number of the additionally generated clusters of fundamental solitons reduces to one, in either case of $\left(S_{1}, S_{2}\right)=(+1,-1)$ and $(+2,-2)$, see Figs. $1(\mathrm{~d})$ and $2(\mathrm{~d})$. Actually, the "unfinished vortices" are long-lived intermediate states, which later split into dipolar pairs of fundamental solitons in both cases of $(+1,-1)$ and $(+2,-2)$, see below.

Taking still larger values of $\chi$, we observe that the collision does not generate any clusters, but only two "unfinished" solitary vortices, as demonstrated in Figs. 1(e) and 2(e). The absence of extra clusters indicates a proximity to a quasielastic collision. Eventually, at high values of $\chi$, the collision becomes elastic, as clearly seen in Figs. 1(f) and $2(\mathrm{f})$. In the latter case, the collision results in a small decrease of the relative velocity of the solitary vortices.

The five collision scenarios identified above are further illustrated by Fig. 3, which shows the evolution of the field in the plane of $(t, z)$ [only for pair $\left(S_{1}, S_{2}\right)=(+2,-2)$, the pictures for $(+1,-1)$ being quite similar]. In fact, this figure displays trajectories of centers of the vortices (including the "unfinished" ones) and soliton clusters before and after the collisions (the intrinsic structure of the densely packed clusters is not visible in the figure). The eventual splitting of the "unfinished vortices" and slow expansion of the clusters occur on an essentially longer scale of the propagation distance, see below.

\section{Post-collision evolution}

The above-mentioned eventual splitting of two- and fourhumped "unfinished" vortices [the ones displayed in Figs. 

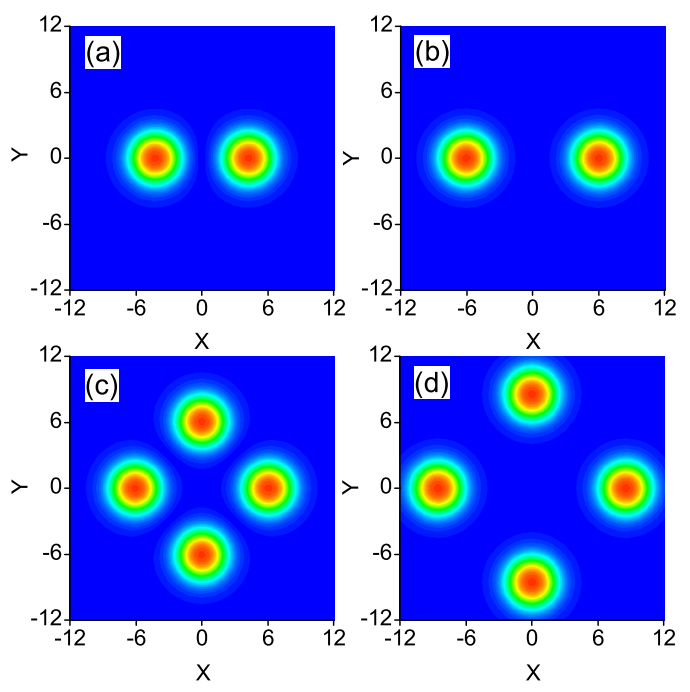

FIG. 5. (Color online) Contour plots of $|U(x, y, t)|^{2}$ at $t=0$ demonstrate slow expansion of the dipole and quadrupole clusters taken from Figs. 1(b) and 2(d), respectively. The dipole cluster is shown at $z=100$ (a) and $z=3700$ (b), and the quadrupole one-at $z=130$ (c) and $z=3700$ (d).

1(d) and 2(d), respectively] into a set of two fundamental solitons, at values of $z$ essentially larger than those corresponding to Figs. 1-3, is shown in Fig. 4.

Dipole and quadrupole clusters of fundamental solitons, which are generated by the inelastic collisions of $(+1,-1)$ and $(+2,-2)$ vortex pairs, respectively, feature very slow expansion in the course of the post-collision evolution, as demonstrated by Fig. 5 (both examples displayed in the figure pertain to the initial kick factor of $\chi=1$ ). The dynamics of the expansion is additionally illustrated in Figs. 6(a) and 6(b) by plots displaying the size of the clusters and their total energy, defined as per Eq. (5), as functions of $z$.

The slowly expanding clusters are very robust objects. In particular, the evolution of the dipolar and quadrupolar clusters (two- and four-soliton ones) under the action of a suddenly applied $10 \%$ white-noise perturbation demonstrates, in Figs. 6(c) and 6(d), quick suppression of the perturbation. The most essential feature providing for the robustness of the clusters is the opposite sign of the field in adjacent solitons, i.e., repulsion between them. In this connection, it is relevant to mention that the dynamics of weakly nonstationary $2 \mathrm{D}$ and 3D soliton clusters was studied in detail, in various conservative and dissipative settings [24-27].

\section{CONCLUSION}

In this work, we aimed to study collisions of counterrotating 3D solitary vortices, with opposite topological charges $\pm S$. To the best of our knowledge, this dynamical process was not considered before, in three-dimensional conservative and dissipative models alike. We used the simplest 3D model which admits stable vortex solitons, viz., the com-
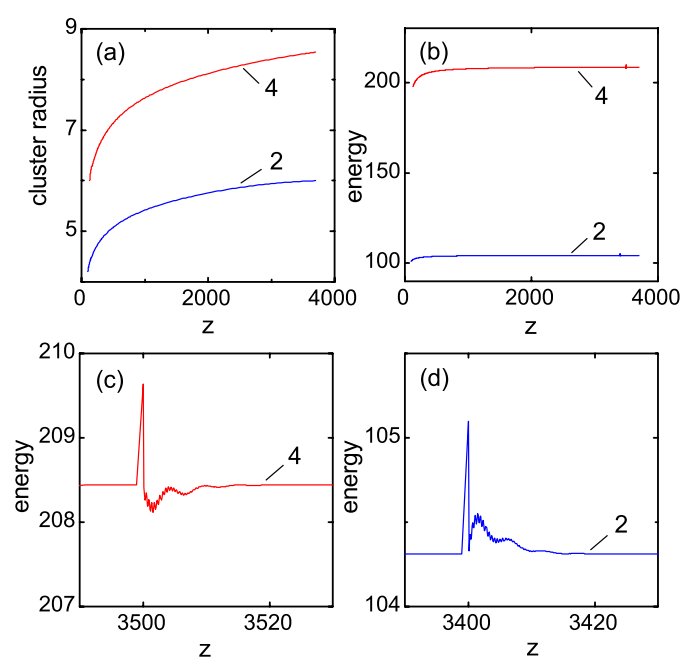

FIG. 6. (Color online) Evolution of the radii of the two-soliton and four-soliton clusters (a) and of their total energies (b) in the course of their slow expansion. Panels (c) and (d) show the evolution of the total energy of the two- and four-soliton clusters, to which strong white-noise perturbation was added at $z=3400$ and $z$ $=3500$, respectively.

plex Ginzburg-Landau equation with a cubic-quintic nonlinearity, which may be realized in terms of laser cavities. The phenomenology of the vortex-antivortex collisions in this model is completely different from what was reported in recent work [19] in collisions between vortices with equal topological charges (in particular, collisions between corotating vortices do not generate soliton clusters). Slow collisions give rise to the merger of the colliding vortices into one or two slowly expanding dipole or quadrupole clusters formed by two or four fundamental solitons, in the cases of $S=1$ and 2 , respectively. Faster collisions generate pairs of counterrotating "unfinished vortices" (in the combination with a soliton cluster or without it). Later, the unfinished vortices split into soliton pairs. The pairs of the "unfinished vortices," as well as dipolar or quadrupolar clusters, if they emerge in pairs, feature counter-rotation, which is subject to gradual braking under the action of the diffusion. Eventually, the collisions become elastic. It is noteworthy that the dipole and quadrupole clusters generated by the collisions are very robust aggregates.

This work, as well as Ref. [19], that deal with the collisions of co-rotating 3D vortex solitons with equal intrinsic vorticities, $(S, S)(S=1$ and 2$)$, report results for coaxial configurations. A challenging problem is the analysis of collisions in a more general geometrical setting. Another generalization, which would be relevant to the models of laser cavities, may be taking into regard the third-order groupvelocity dispersion, as was done, in the context of the twodimensional complex Ginzburg-Landau equation with the cubic-quintic nonlinearity, in Ref. [28].

\section{ACKNOWLEDGMENT}

This work was supported, in part, by Deutsche Forschungsgemeinschaft (DFG), Bonn, Germany. 
[1] I. S. Aranson and L. Kramer, Rev. Mod. Phys. 74, 99 (2002); B. A. Malomed, in Encyclopedia of Nonlinear Science, edited by A. Scott (Routledge, New York, 2005), 157.

[2] N. N. Rosanov, Spatial Hysteresis and Optical Patterns (Springer, Berlin, 2002).

[3] J. Lega, J. V. Moloney, and A. C. Newell, Phys. Rev. Lett. 73, 2978 (1994); Physica D 83, 478 (1995).

[4] S. Barland, J. R. Tredicce, M. Brambilla, L. A. Lugiato, S. Balle, M. Giudici, T. Maggipinto, L. Spinelli, G. Tissoni, T. Knodl, M. Miller, and R. Jager, Nature (London) 419, 699 (2002); Z. Bakonyi, D. Michaelis, U. Peschel, G. Onishchukov, and F. Lederer, J. Opt. Soc. Am. B 19, 487 (2002); E. A. Ultanir, G. I. Stegeman, D. Michaelis, C. H. Lange, and F. Lederer, Phys. Rev. Lett. 90, 253903 (2003); N. N. Rosanov, S. V. Fedorov, and A. N. Shatsev, ibid. 95, 053903 (2005).

[5] W. J. Firth and D. V. Skryabin, Phys. Rev. Lett. 79, 2450 (1997).

[6] B. A. Malomed, D. Mihalache, F. Wise, and L. Torner, J. Opt. B: Quantum Semiclassical Opt. 7, R53 (2005).

[7] A. S. Desyatnikov, Y. S. Kivshar, and L. Torner, Prog. Opt. 47, 291 (2005).

[8] V. I. Petviashvili and A. M. Sergeev, Dokl. Akad. Nauk SSSR 276, 1380 (1984) [Sov. Phys. Dokl. 29, 493 (1984)].

[9] B. A. Malomed, Physica D 29, 155 (1987); O. Thual and S. Fauve, J. Phys. (Paris) 49, 1829 (1988); S. Fauve and O. Thual, Phys. Rev. Lett. 64, 282 (1990); W. van Saarloos and P. C. Hohenberg, ibid. 64, 749 (1990); V. Hakim, P. Jakobsen, and Y. Pomeau, Europhys. Lett. 11, 19 (1990); B. A. Malomed and A. A. Nepomnyashchy, Phys. Rev. A 42, 6009 (1990); P. Marcq, H. Chaté, and R. Conte, Physica D 73, 305 (1994); N. Akhmediev and V. V. Afanasjev, Phys. Rev. Lett. 75, 2320 (1995); H. R. Brand and R. J. Deissler, ibid. 63, 2801 (1989); R. J. Deissler and H. R. Brand, ibid. 72, 478 (1994); 74, 4847 (1995); 81, 3856 (1998); V. V. Afanasjev, N. Akhmediev, and J. M. Soto-Crespo, Phys. Rev. E 53, 1931 (1996); J. M. SotoCrespo, N. Akhmediev, and A. Ankiewicz, Phys. Rev. Lett. 85, 2937 (2000); W. Chang, A. Ankiewicz, and N. Akhmediev, Phys. Lett. A 362, 31 (2007).

[10] F. Smektala, C. Quemard, V. Couderc, and A. Barthélémy, J. Non-Cryst. Solids 274, 232 (2000); G. Boudebs, S. Cherukulappurath, H. Leblond, J. Troles, F. Smektala, and F. Sanchez, Opt. Commun. 219, 427 (2003).

[11] C. Zhan, D. Zhang, D. Zhu, D. Wang, Y. Li, D. Li, Z. Lu, L. Zhao, and Y. Nie, J. Opt. Soc. Am. B 19, 369 (2002).

[12] G. S. Agarwal and S. Dutta Gupta, Phys. Rev. A 38, 5678 (1988).

[13] E. L. Falcão-Filho, C. B. de Araújo, and J. J. Rodrigues, Jr., J. Opt. Soc. Am. B 24, 2948 (2007).
[14] R. A. Ganeev, M. Baba, M. Morita, A. I. Ryasnyansky, M. Suzuki, M. Turu, and H. Kuroda, J. Opt. A, Pure Appl. Opt. 6, 282 (2004).

[15] L.-C. Crasovan, B. A. Malomed, and D. Mihalache, Phys. Rev. E 63, 016605 (2001); Phys. Lett. A 289, 59 (2001).

[16] D. Mihalache, D. Mazilu, F. Lederer, Y. V. Kartashov, L.-C. Crasovan, L. Torner, and B. A. Malomed, Phys. Rev. Lett. 97, 073904 (2006).

[17] D. Mihalache, D. Mazilu, F. Lederer, H. Leblond, and B. A. Malomed, Phys. Rev. A 75, 033811 (2007); 76, 045803 (2007).

[18] J. M. Soto-Crespo, N. Akhmediev, and P. Grelu, Phys. Rev. E 74, 046612 (2006); N. Akhmediev, J. M. Soto-Crespo, and P. Grelu, Chaos 17, 037112 (2007).

[19] D. Mihalache, D. Mazilu, F. Lederer, H. Leblond, and B. A. Malomed, Phys. Rev. A 77, 033817 (2008).

[20] P. Grelu, J. M. Soto-Crespo, and N. Akhmediev, Opt. Express 13, 9352 (2005); J. M. Soto-Crespo, P. Grelu, and N. Akhmediev, ibid. 14, 4013 (2006); V. Skarka and N. B. Aleksić, Phys. Rev. Lett. 96, 013903 (2006).

[21] A. Desyatnikov, A. Maimistov, and B. Malomed, Phys. Rev. E 61, 3107 (2000); D. Mihalache, D. Mazilu, L.-C. Crasovan, B. A. Malomed, and F. Lederer, ibid. 61, 7142 (2000); D. Mihalache, D. Mazilu, L. C. Crasovan, I. Towers, A. V. Buryak, B. A. Malomed, L. Torner, J. P. Torres, and F. Lederer, Phys. Rev. Lett. 88, 073902 (2002).

[22] H. Sakaguchi, Physica D 210, 138 (2005)

[23] E. A. Ultanir, G. I. Stegeman, C. H. Lange, and F. Lederer, Opt. Lett. 29, 283 (2004).

[24] M. Soljacic, S. Sears, and M. Segev, Phys. Rev. Lett. 81, 4851 (1998)

[25] A. S. Desyatnikov and Yu. S. Kivshar, Phys. Rev. Lett. 88, 053901 (2002).

[26] Y. V. Kartashov, L.-C. Crasovan, D. Mihalache, and L. Torner, Phys. Rev. Lett. 89, 273902 (2002); D. Mihalache, D. Mazilu, L.-C. Crasovan, B. A. Malomed, F. Lederer, and L. Torner, Phys. Rev. E 68, 046612 (2003); L.-C. Crasovan, Y. V. Kartashov, D. Mihalache, L. Torner, Y. S. Kivshar, and V. M. Perez-Garcia, ibid. 67, 046610 (2003); D. Mihalache, D. Mazilu, L.-C. Crasovan, B. A. Malomed, F. Lederer, and L. Torner, J. Opt. B: Quantum Semiclassical Opt. 6, S333 (2004); L.-C. Crasovan, G. Molina-Terriza, J. P. Torres, L. Torner, V. M. Perez-Garcia, and D. Mihalache, Phys. Rev. E 66, 036612 (2002).

[27] D. V. Skryabin and A. G. Vladimirov, Phys. Rev. Lett. 89, 044101 (2002)

[28] H. Sakaguchi and B. A. Malomed, Physica D 159, 91 (2001). 